This item was submitted to Loughborough's Research Repository by the author.

Items in Figshare are protected by copyright, with all rights reserved, unless otherwise indicated.

\title{
Rendezvous and standoff target tracking guidance using differential geometry
}

PLEASE CITE THE PUBLISHED VERSION

http://dx.doi.org/10.1007/s10846-012-9751-0

\section{PUBLISHER}

(C) Springer Science+Business Media B.V.

\section{VERSION}

AM (Accepted Manuscript)

\section{PUBLISHER STATEMENT}

This work is made available according to the conditions of the Creative Commons Attribution-NonCommercialNoDerivatives 4.0 International (CC BY-NC-ND 4.0) licence. Full details of this licence are available at: https://creativecommons.org/licenses/by-nc-nd/4.0/

\section{LICENCE}

CC BY-NC-ND 4.0

\section{REPOSITORY RECORD}

Oh, Hyondong, Seungkeun Kim, Hyo-Sang Shin, Brian A. White, Antonios Tsourdos, and Camille A. Rabbath. 2015. "Rendezvous and Standoff Target Tracking Guidance Using Differential Geometry". figshare. https://hdl.handle.net/2134/17854. 


\title{
Rendezvous and Standoff Target Tracking Guidance Using Differential Geometry
}

\author{
Hyondong $\mathrm{Oh}^{1}$, Seungkeun Kim${ }^{2}$, Hyo-Sang $\operatorname{Shin}^{1}$, Brian A. White ${ }^{1}$, \\ Antonios Tsourdos ${ }^{1}$, and Camille Alain Rabbath ${ }^{3}$
}

\begin{abstract}
This paper proposes UAV rendezvous and standoff tracking guidance laws against a moving target using differential geometry. Searching and subsequent tracking of moving ground based target is one of the primary capabilities of cooperative UAVs. In performing such missions, UAVs are to approach a target and keep a certain distance, known as a standoff distance. This allows target tracking without being noticed and acquisition of accurate target information. In this study, standoff target tracking is proposed using the solution of differential geometry between the UAV and the target. The proposed algorithm brings several advantages along with its inherent simplicity: rigorous stability, explicit use of a target velocity, and tuning parameter reduction. The feasibility and performance of the proposed approach is not only mathematically analysed, but also verified through realistic scenarios.
\end{abstract}

\section{INTRODUCTION}

The large scale of UAV (Unmanned Aerial Vehicle) applications has proliferated vastly within the last two decades. The operational experience of UAVs has proven that their technology can bring a dramatic impact to both military and civilian applications. This includes, but not limited to: obtaining real-time, relevant situational awareness before making contact; helping operators to lead appropriate decision making; and reducing risk to the mission and operation.

\footnotetext{
${ }^{1}$ Hyondong Oh, Hyo-Sang Shin, Antonios Tsourdos and Brian A. White are with the Department of Informatics and Systems Engineering, Cranfield University, Defence Academy of the UK, Swindon, SN6 8LA, United Kingdom, e-mail: \{h.oh, h.shin, b.a.white, a.tsourdos\}@cranfield.ac.uk

${ }^{2}$ Seungkeun Kim is with the Department of Aerospace Engineering, Chungnam National University, 99 Daehak-ro, Yuseong-gu, Daejeon 305-764, Republic of Korea, e-mail: skim78@cnu.ac.kr.

${ }^{3}$ Camille Alain Rabbath is with the Precision Weapons Section, Defence Research and Development Canada Valcartier, 2459 Boul. Pie XI North Quebec, QC, Canada, G3J 1X5, e-mail: CamilleAlain.Rabbath@drdc-rddc.gc.ca
}

Groups of UAVs are of special interest due to their ability to coordinate simultaneous coverage of large areas, or co-operate to achieve common goals. Specific applications under consideration for groups of cooperating UAVs are border patrol, search and rescue, surveillance, airborne police enforcement, adversarial or enemy monitoring, and ground vehicle convoy. In these applications, searching and subsequent target tracking become one of key UAV capabilities required.

For search and tracking of ground based target, it is desirable to acquire accurate target motion information, as well as to track the target without being noticed. These capabilities can be enabled by approaching the target and keeping a certain relative distance with a prescribed inter-vehicle angular separation. The certain relative distance from the target is called the standoff distance and therefore this approach is known as standoff target tracking. A configuration of UAVs for standoff tracking generally adopts two strategies: i) distribute UAVs to have equal angular separation around the target [1] or ii) position them at a certain angular position in the orbit. In the second strategy, the number of team members and sensor characteristics are taken into account to determine the angular separation maximising the estimation accuracy of target information [2], [3].

In a domain of standoff target tracking, Lawrence [4] firstly proposed the application of Lyapunov vector field, which was further investigated by Frew et. al. [5], [6] to incorporate phase-keeping as well as standoff tracking for two-UAV formation. They proposed a decoupled guidance structure in which speed and heading-rate are separately controlled for standoff distance and phase angle keeping, respectively. Summers et. al. [7] extended this phase-keeping idea to multiple 
UAVs using information architectures in vehicle formations. Similarly, Kingston et. al. [8] used vector field approach, however, they introduced a sliding mode control concept and orbit radius change without velocity change for phase-keeping of multiple UAVs. Chen et. al. [9] proposed the use of a tangent-plus-Lyapunov vector field which includes a simple switching logic between tangent and Lyapunov vector fields to make convergence to the standoff circle faster. Moreover, Sepulchre et. al. [10] applied the collective control of multiagent system to stabilise circular formation around the target. Klein and Morgansen [11] proposed a steering control law making the velocity of the collective centroid match a reference velocity allowing tracking of a moving target. Wise and Rysdyk [12] well surveyed and compared the different methodologies and recently, Prevost et. al. [13] and Kim et. al. [14] applied a model-based predictive control for standoff tracking.

This paper proposes UAV rendezvous and standoff tracking guidance to a moving ground target using differential geometry motivated by previous works [15], [16]. Using the relative geometry, convergent, divergent and parallel solutions can be obtained depending on their initial positions and the velocity ratio between them. Then, the convergent solution can be used to guide the UAV on the corresponding rendezvous geometry associated with the target movement. In a similar way, a novel guidance law for standoff tracking is derived by superimposing a standoff orbit circle around the target position. In order to verify the feasibility and benefits of the proposed approach, numerical simulations are performed using a realistic scenario, in which two cooperative UAVs are equipped with MTIR (Moving Target Indicator Radar) and run extended Information filter with decentralised sensor fusion for target localisation.

The proposed differential geometric guidance law has several advantages along with its inherent simplicity over the other standoff tracking guidance laws. First of all, whilst most of literatures have focused on the stability analysis limited to standoff tracking of a fixed target, the proposed approach can analyse stability for both rendezvous and standoff tracking of a moving target. Another benefit is that the guidance command can explic- itly consider a target velocity for enhancing the tracking performance when its estimation by the localisation filter is reasonably accurate. Lastly, the proposed guidance law requires the reduced number of tuning variables, only a curvature command, unlike other approaches such as vector field guidance requiring more parameters for appropriate vector field generation as well as guiding vehicle into the field [8], [17].

The remainder of this paper is organised as follows. Section II introduces rendezvous geometry between the UAV and the target using differential geometry. The description of standoff tracking geometry follows in Section III. Section IV proposes the guidance algorithms to generate the UAV turnrate command for both rendezvous and standoff tracking against a moving target and then proves its global convergence using Lyapunov theory. Lastly, numerical simulations are performed to verify the performances of the proposed methodology in Section V. Conclusions and future works are given in Section VI.

\section{Differential Geometry ASSOCiATED WITH UAV AND TARGET}

\section{A. Rendezvous geometry}

Consider an UAV and a moving target with their motion associated each other in a Frenet-Serret frame [18], [15]. Rendezvous geometry is built up using the two-dimensional Frenet-Serret frame defined by a tangent vector $\mathbf{t}$ and a normal vector $\mathbf{n}$ of each vehicle as shown in Fig. 1. The required

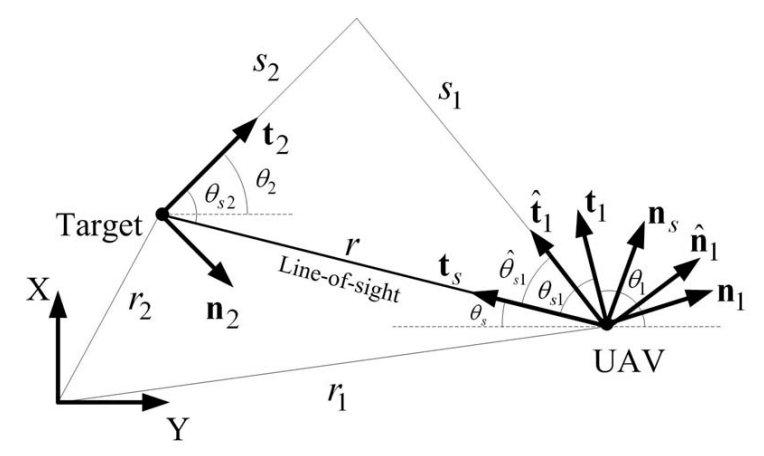

Fig. 1. Guidance geometry

tangent direction of the UAV for rendezvous, $\hat{\mathbf{t}}_{1}$, is represented with the following vector addition on the rendezvous triangle in Fig. 1:

$$
s_{1} \hat{\mathbf{t}}_{1}=r \mathbf{t}_{s}+s_{2} \mathbf{t}_{2}
$$


where $\mathbf{t}_{2}$ and $\mathbf{t}_{s}$ are a current velocity vector of the target and a line-of-sight (LOS) vector from the UAV to the target, respectively, $r$ is a distance between the UAV and the target, and $s_{1}$ and $s_{2}$ are the resultant lengths of the tangent vectors to the rendezvous point. Let us define a velocity ratio of the UAV and the target, $\gamma$, as:

$$
\gamma=\frac{v_{1}}{v_{2}}=\frac{s_{1}}{s_{2}}
$$

where $v_{i}$ is the constant speed of each vehicle. Then, Eq. (1) changes to:

$$
\hat{\mathbf{t}}_{1}=\frac{1}{\gamma}\left[\frac{r}{s_{2}} \mathbf{t}_{s}+\mathbf{t}_{2}\right] \text {. }
$$

Applying a cosine rule to the geometry around the LOSs to the target gives:

$$
\left(\frac{r}{s_{2}}\right)^{2}-2 \cos \left(\theta_{s 2}\right)\left(\frac{r}{s_{2}}\right)-\left(\gamma^{2}-1\right)=
$$

Solving this equation gives:

$$
\left(\frac{r}{s_{2}}\right)=\cos \left(\theta_{s 2}\right) \pm \sqrt{\gamma^{2}-\sin ^{2}\left(\theta_{s 2}\right)} .
$$

For this case of a straight line rendezvous, the triangle of the rendezvous point and vehicle positions is thus invariant in shape, but will shrink or grow as UAVs travel along the solution. One solution will result in rendezvous, the other in a divergent geometry as shown in Figure 2. In this figure, a

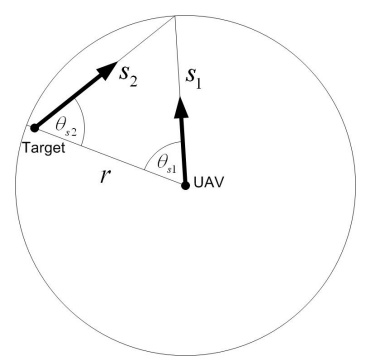

(a) Convergent Solution

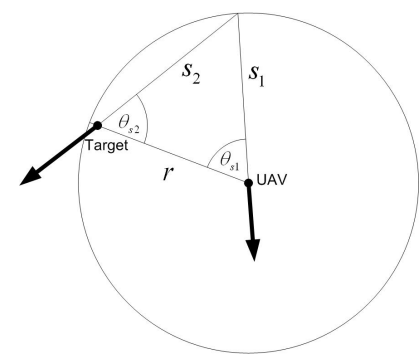

(b) Divergent Solution
Fig. 2. Solution geometries for rendezvous

circle with radius of $s_{1}$ is drawn to visualise the reachable area of the UAV considering the length (or velocity) ratio $\gamma=\frac{s_{1}}{s_{2}}$ as in Eq. (2), and possible initial conditions for rendezvous geometry between the UAV and target. The convergent solution uses the positive square root, whilst the divergent solution uses the negative square root. Hence for this case, the solution will always use the positive square root, as:

$$
\left(\frac{r}{s_{2}}\right)=\cos \left(\theta_{s 2}\right)+\sqrt{\gamma^{2}-\sin ^{2}\left(\theta_{s 2}\right)} .
$$

It is also worth exploring the solution as a function of the speed ratio $\gamma$. If $\gamma>1$, then the expression within the square root is always greater than zero for all $\theta_{s 2}$, or:

$$
\gamma^{2}-\sin ^{2}\left(\theta_{s 2}\right)>0, \quad \gamma>1
$$

This means that there will always be a real solution for any initial geometry. Such a condition is shown in Fig. 3(a). However, if $\gamma<1$, then there will be

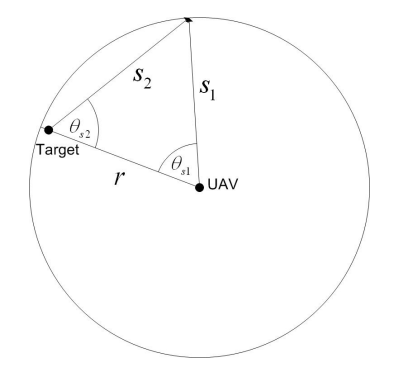

(a) Rendezvous solution always possible

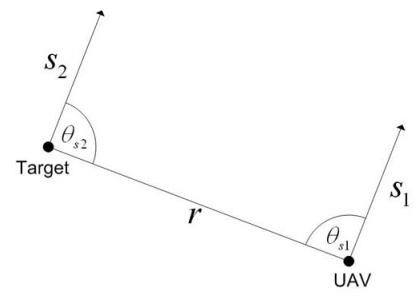

(c) Parallel solution
Fig. 3. Rendezvous solutions depending on initial positions and $\gamma$

some $\theta_{s 2}$ for which:

$$
\gamma^{2}-\sin ^{2}\left(\theta_{s 2}\right)<0, \quad \gamma<1
$$

This implies that for some geometry, a real solution is not possible, and the UAV will not be able to rendezvous with the target. For example, for the fixed target direction shown in Fig. 3(b), the circle indicating all the possible travel directions of the UAV has no intersection with the trajectory of the target abiding by the length ratio $\gamma$, and thus there is no solution. For the case of the two vehicles having the same speed, i.e. $\gamma=1$, the rendezvous geometry equation now becomes:

$$
\hat{\mathbf{t}}_{1}=\left[\frac{r}{s_{2}} \mathbf{t}_{s}+\mathbf{t}_{2}\right]
$$


with

$$
\left(\frac{r}{s_{2}}\right)^{2}-2 \cos \left(\theta_{s 2}\right)\left(\frac{r}{s_{2}}\right)=0 .
$$

The solution for this case is given by:

$$
\left(\frac{r}{s_{2}}\right)=2 \cos \left(\theta_{s 2}\right) \text { or } 0 .
$$

There is no longer an imaginary solution, and now two real solutions are given. The first solution implies a geometry which gives rise to an isosceles triangle solution as before, but only where $s_{1}=s_{2}$. Since the solution depends on the initial positions and orientation of the target, global convergence is not possible for this case. The second solution implies that the ratio $r / s_{2}$ is zero. For $r>0$, this implies $s_{2}=\infty$ or $\theta_{s 1}=\theta_{s 2}=\pi / 2$. This solution results in the UAV and the target moving on parallel courses that neither converge or diverge as shown in Fig. 3(c), and the vehicles will maintain the geometry both in shape and size for this solution. Hence this solution is of interest when the vehicles are required to move in some form of group or formation where they will retain some sort of cohesion in manoeuvre. Note that this condition does not imply any particular geometry, much as the other solutions do. All that is required is that the ratio of the two paths $s_{1}$ and $s_{2}$ in the solution are the same as the speed ratio between them.

The guidance algorithm must therefore compute the required velocity vector tangent $\hat{\mathbf{t}}_{1}$ using Eq. (3) along with Eq. (5) for the UAV and produce a closed loop system which drives the current tangent vector $\mathbf{t}_{1}$ onto it in a stable manner.

\section{B. Standoff tracking geometry}

This section extends the rendezvous problem of the previous section into standoff tracking for which the UAV needs to track the moving target while maintaining a certain distance from it. In a similar way to the previous section, let us consider the associated geometry of the UAV and the target with newly adopting a standoff distance $d_{m}$. The relative velocity of the UAV with respect to the target is shown in Fig. 4 for rendezvous. The relative velocity of the UAV with respect to the target is given by:

$$
\mathbf{v}_{r}=\mathbf{v}_{1}-\mathbf{v}_{2}
$$

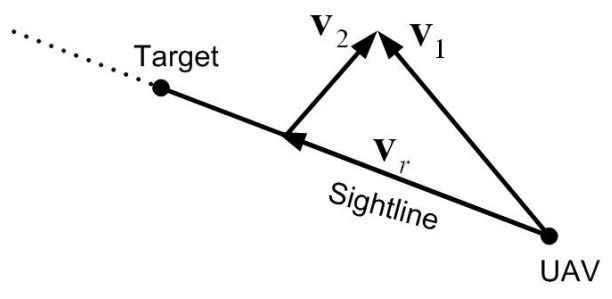

Fig. 4. Relative velocity for rendezvous

The condition for rendezvous is shown to be such that the relative velocity vector should lie along the LOS between the UAV and the target. This ensures that the geometry does not change over time as the rendezvous triangle shrinks but maintains its shape. This is consistent with the fact that the solution of $r / s$ is constant as defined in Eq. (5). This can now be modified to produce the geometry of standoff tracking by superimposing a circle of radius $d_{m}$ around the target as shown in Fig. 5. If the relative velocity vector $\mathbf{v}_{r}$ is aligned with

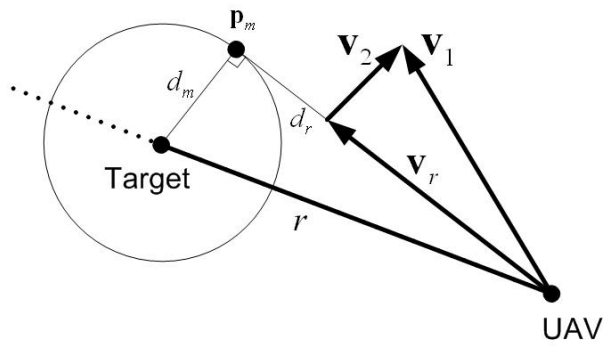

Fig. 5. Relative velocity for standoff tracking orbit approach

the tangent line to the standoff circle, then the rendezvous point for standoff tracking will be at $\mathbf{p}_{\mathbf{m}}$.

Now, the rendezvous geometry shown in Fig. 1 is modified to fit the standoff tracking as shown in Fig. 6. Herein, the original rendezvous triangle is modified into the triangle given by $\left\{\mathbf{p}_{\mathbf{1}}, \mathbf{p}_{\mathbf{m}}, \mathbf{p}_{\mathbf{s}}\right\}$, for both a clockwise and anti-clockwise rotation from the LOS to $\mathbf{p}_{\mathbf{m}}$. The vector sum on the engagement geometry is given as:

$$
\hat{\mathbf{t}}_{1}=\frac{1}{\gamma}\left[\frac{d_{r}}{s_{2}} \mathbf{t}_{d}+\mathbf{t}_{2}\right]
$$

where $\mathbf{t}_{d}$ is the unit tangent vector from the UAV to $\mathbf{p}_{\mathbf{m}}$, and $d_{r}$ is the distance between the UAV and rendezvous point $\mathbf{p}_{\mathbf{m}}$. Applying the cosine rule to 


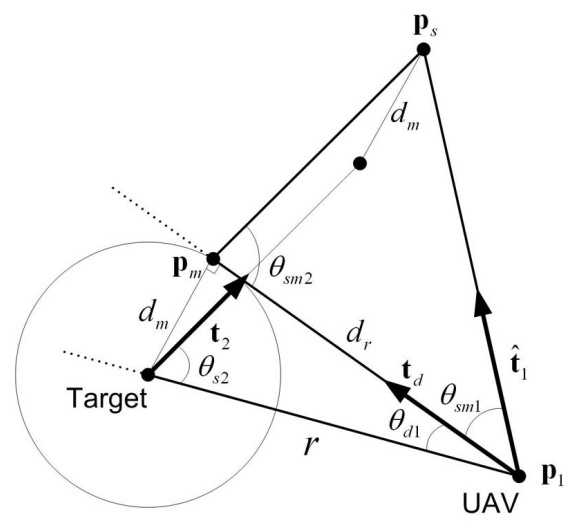

(a) Clockwise solution

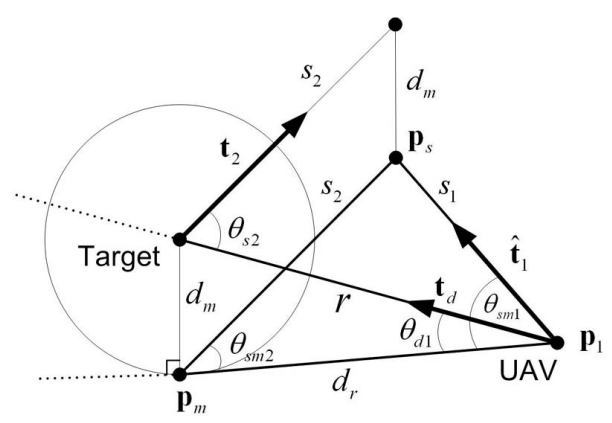

(b) Anti-clockwise solution

Fig. 6. Geometry for standoff tracking

this geometry gives:

$$
\left(\frac{d_{r}}{s_{2}}\right)^{2}-2 \cos \left(\theta_{s m 2}\right)\left(\frac{d_{r}}{s_{2}}\right)-\left(\gamma^{2}-1\right)=0
$$

where

$$
\begin{aligned}
d_{r} & =\sqrt{r^{2}-d_{m}^{2}} \\
\theta_{s m 2} & =\theta_{s 2} \pm \theta_{d 1} .
\end{aligned}
$$

Herein, $\theta_{d 1}$ is either added for a clockwise solution and subtracted for an anti-clockwise solution. Substituting the following relations:

$$
\begin{aligned}
\cos \left(\theta_{d 1}\right) & =\frac{d_{r}}{r}=\frac{\sqrt{r^{2}-d_{m}^{2}}}{r} \\
\sin \left(\theta_{d 1}\right) & =\frac{d_{m}}{r}
\end{aligned}
$$

into

$$
\begin{array}{r}
\cos \left(\theta_{s m 2}\right)=\cos \left(\theta_{s 2} \pm \theta_{d 1}\right) \\
=\cos \left(\theta_{s 2}\right) \cos \left(\theta_{d 1}\right) \mp \sin \left(\theta_{s 2}\right) \sin \left(\theta_{d 1}\right)
\end{array}
$$

gives

$$
\cos \left(\theta_{s m 2}\right)=\frac{\sqrt{r^{2}-d_{m}^{2}}}{r} \cos \left(\theta_{s 2}\right) \mp \frac{d_{m}}{r} \sin \left(\theta_{s 2}\right) .
$$

Note that as the standoff distance is reached, the parameters become:

$$
r \rightarrow d_{m}, d_{r} \rightarrow 0, \theta_{d 1} \rightarrow \frac{\pi}{2}
$$

Although the geometry of this case is not fixed with respect to the LOS, the solution requires the relative velocity vector to lie along the tangent line vector $\mathbf{t}_{d}$ which will not be changed, and the two vehicle velocity vectors are fixed. Therefore, the triangle $\left\{\mathbf{p}_{\mathbf{1}}, \mathbf{p}_{\mathbf{m}}, \mathbf{p}_{\mathbf{s}}\right\}$ is fixed in shape and orientation, and will shrink as the UAV approaches to the standoff orbit $\mathbf{p}_{\mathbf{m}}$. Then, the ratio $d_{r} / s_{2}$, a solution to Eq. (14), will have a fixed solution as:

$$
\left(\frac{d_{r}}{s_{2}}\right)=\cos \left(\theta_{s m 2}\right) \pm \sqrt{\gamma^{2}-\sin ^{2}\left(\theta_{s m 2}\right)} .
$$

The guidance algorithm must therefore compute the required velocity vector $\hat{\mathbf{t}}_{1}$ using Eq. (13) along with Eq. (21) for the UAV and produce a closed loop system which drives the current tangent vector $\mathbf{t}_{1}$ onto it in a stable manner, as in the case for rendezvous.

\section{UAV GUIDANCE LAW FOR RENDEZVOUS AND STANDOFF TRACKING}

This section first introduces UAV dynamic model and then proposes the guidance laws for both rendezvous and standoff tracking case. For ease of deriving the guidance laws, this paper assumes that at the current sampling time, the target is instantaneously non-manoeuvring but its velocity is exploited, which can be estimated by tracking filters of the UAVs.

\section{A. UAV dynamic model}

Assuming each UAV has a low-level flight controller such as SAS (Stability Augmentation System) and CAS (Controllability Augmentation System) for heading and velocity hold functions, this study aims to design guidance inputs to this low-level controller for rendezvous and standoff target tracking. Consider a two-dimensional UAV kinematic model [14] as:

$$
\left(\begin{array}{c}
\dot{x} \\
\dot{y} \\
\dot{\psi} \\
\dot{v} \\
\dot{\omega}
\end{array}\right)=f(\mathbf{x}, \mathbf{u})=\left(\begin{array}{c}
v \cos \psi \\
v \sin \psi \\
\omega \\
-\frac{1}{\tau_{v}} v+\frac{1}{\tau_{1}} u_{v} \\
-\frac{1}{\tau_{\omega}} \omega+\frac{1}{\tau_{\omega}} u_{\omega}
\end{array}\right)
$$


where $\mathbf{x}=(x, y, \psi, v, \omega)^{T}$ are the inertial position, heading, speed and yaw rate of the UAV, respectively. $\tau_{v}$ and $\tau_{\omega}$ are time constants for considering actuator delay. $\mathbf{u}=\left(u_{v}, u_{\omega}\right)^{T}$ are the commanded speed and turning rate constrained by the following dynamic limits of a fixed-wing UAV:

$$
\begin{aligned}
\left|u_{v}-v_{0}\right| & \leq v_{\max } \\
\left|u_{\omega}\right| & \leq \omega_{\max }
\end{aligned}
$$

where $v_{0}$ is a nominal speed of UAV. The continuous UAV model in Eq. (22) can be discretised by Euler integration into:

$$
\mathbf{x}_{k+1}=f_{d}\left(\mathbf{x}_{k}, \mathbf{u}_{k}\right)=\mathbf{x}_{k}+T_{s} f\left(\mathbf{x}_{k}, \mathbf{u}_{k}\right)
$$

where $\mathbf{x}_{k}=\left(x_{k}, y_{k}, \psi_{k}, v_{k}, \omega_{k}\right)^{T}, \quad \mathbf{u}_{k}=$ $\left(u_{v k}, u_{\omega k}\right)^{T}$, and $T_{s}$ is a sampling time.

\section{B. Rendezvous case}

This section designs a guidance law for rendezvous covering all the convergent solutions discussed in Section II. Assuming the UAV has a speed advantage over the target, the positive solutions are considered from Eq. (4). Let us define the angle between the required tangent vector $\hat{\mathbf{t}}_{1}$ and the current UAV tangent vector $\mathbf{t}_{1}$ as:

$$
\chi_{1}=\left(\pi-\theta_{s 1}\right)-\hat{\theta}_{s 1}
$$

where $\left(\pi-\theta_{s 1}\right)$ and $\hat{\theta}_{s 1}$ are the current UAV tangent angle and the required tangent angle with respect to the LOS, respectively, as shown in Fig. 1. To guide the UAV onto the required geometry, considering an actively rotating tangent vector $\mathbf{t}_{1}$, the guidance command $u_{\omega}$ for turn rate is set by using a curvature command as:

$$
u_{\omega}=\dot{\theta}_{1}=\kappa_{1} v_{1}
$$

where $v_{1}$ is the UAV speed, and $\kappa_{1}$ is the curvature command. For ease of analysis, the turn rate $\dot{\theta}_{1}$ will be used rather than the explicit $\kappa$ command.

To develop the guidance algorithm and assess the resulting stability, consider a Lyapunov function as:

$$
L_{1}=\frac{1}{2} \chi_{1}^{2}
$$

Its time derivative is given by:

$$
\dot{L}_{1}=\chi_{1} \dot{\chi}_{1}
$$

where

$$
\dot{\chi}_{1}=-\dot{\theta}_{s 1}-\dot{\hat{\theta}}_{s 1} .
$$

The differential of the required angle $\hat{\theta}_{s 1}$ is obtained from examining the rate of change of $\hat{\mathbf{t}}_{1}$ as:

$$
\hat{\mathbf{t}}_{1}=\frac{1}{\gamma}\left[\left(\frac{r}{s_{2}}\right) \mathbf{t}_{s}+\mathbf{t}_{2}\right]
$$

Differentiating this gives:

$$
\dot{\hat{\mathbf{t}}}_{1}=\frac{1}{\gamma}\left[\frac{d}{d t}\left(\frac{r}{s_{2}}\right) \mathbf{t}_{s}+\left(\frac{r}{s_{2}}\right) \dot{\mathbf{t}}_{s}\right]=\dot{\hat{\theta}}_{1} \hat{\mathbf{n}}_{1}
$$

where $\hat{\mathbf{n}}_{1}$ is the normal vector to $\hat{\mathbf{t}}_{1}$, and $\dot{\mathbf{t}}_{2}=0$ is used from the assumption a target is instantaneously non-manoeuvring at the current sampling time. A geometric interpretation of Eq. (31) is reproduced in Fig. 7 to make use of the relation between corresponding angles (especially for $\theta_{s 2}$ and $\left.\hat{\theta}_{s 1}\right)$ intuitively. As the value of $r / s_{2}$ exists between its maximum $\left(1+\gamma\right.$ at $\left.\theta_{s 2}=0\right)$ and minimum $\left(1-\gamma\right.$ at $\left.\theta_{s 2}=\pi\right)$ bounds from Eq. (6), the magnitude of vector $\mathbf{t}_{s}, r /\left(\gamma s_{2}\right)$, has its maximum and minimum values. Figure 7 shows

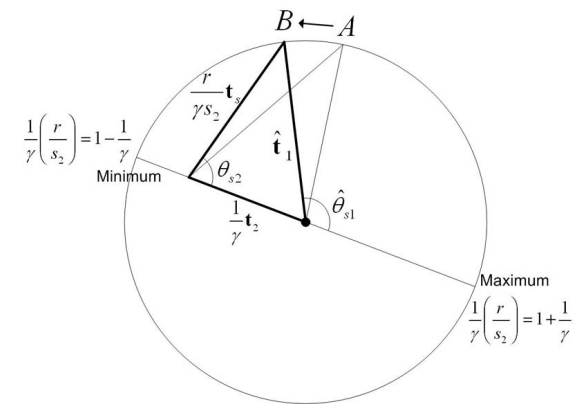

(a) Geometry $\gamma>1$

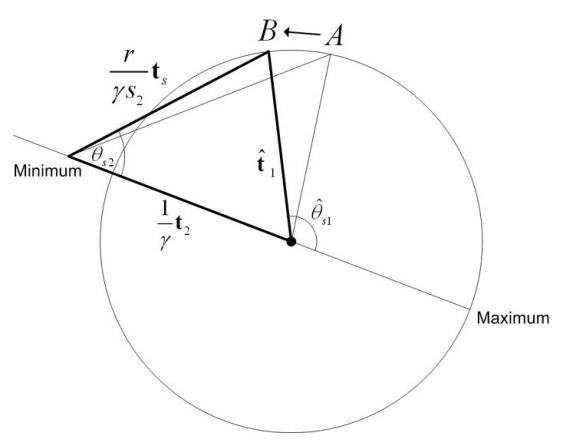

(b) Geometry $\gamma<1$

Fig. 7. Geometric interpretation of rendezvous solutions

that as the engagement geometry changes by the guidance, the solution $\hat{\mathbf{t}}_{1}$ will change and rotate around the circle, and the rotation of the solution 
vector $\hat{\mathbf{t}}_{1}$ is related to the rotation of the LOS vector $\mathbf{t}_{s}$. As shown in Fig. 7, as solution A moves to solution $\mathrm{B}$ when $\gamma>1, \hat{\theta}_{s 1}$ as well as the target to LOS angle $\theta_{s 2}$ increases, and hence there is a monotonic relationship between these two angles. Meanwhile, for the $\gamma<1$ case, the angles oscillates between maximum and minimum bounds according to the condition from Eq. (6). Hence, the following equation holds:

$$
\dot{\hat{\theta}}_{s 1}=\lambda_{1}\left(r / s_{2}, \theta_{s 2}\right) \dot{\theta}_{s 2}
$$

From Fig. 1 and Fig. 7, the relations between the angles are given as:

$$
\begin{aligned}
& \dot{\theta}_{s 2}=\dot{\theta}_{s} \\
& \dot{\hat{\theta}}_{s 1}=\dot{\hat{\theta}}_{1}+\dot{\theta}_{s}
\end{aligned}
$$

since

$$
\begin{aligned}
& \theta_{s 2}=\theta_{2}+\theta_{s} \\
& \hat{\theta}_{s 1}=\theta_{s}+\hat{\theta}_{1}
\end{aligned}
$$

and $\dot{\theta}_{2}=0$. Combining Eq. (33) and Eqs. (34) (35) gives:

$$
\dot{\hat{\theta}}_{s 1}=\dot{\hat{\theta}}_{1}+\dot{\theta}_{s}=\lambda_{1}\left(r / s_{2}, \theta_{s 2}\right) \dot{\theta}_{s} .
$$

Rephrasing this yields:

$$
\dot{\hat{\theta}}_{1}=-\left[1-\lambda_{1}\left(r / s_{2}, \theta_{s 2}\right)\right] \dot{\theta}_{s} .
$$

The time derivative of Lyapunov candidate function in Eq. (30) can be rephrased by using Eq. (39) as:

$$
\dot{L}_{1}=\chi_{1}\left[\dot{\theta}_{1}+\left(1-\lambda_{1}\right) \dot{\theta}_{s}\right] .
$$

since

$$
\dot{\chi}_{1}=-\dot{\theta}_{s 1}-\dot{\hat{\theta}}_{s 1}=\dot{\theta}_{1}+\dot{\theta}_{s}-\left(\dot{\hat{\theta}}_{1}+\dot{\theta}_{s}\right)=\dot{\theta}_{1}-\dot{\hat{\theta}}_{1}
$$

where

$$
\theta_{s 1}=\left(\pi-\theta_{1}\right)-\theta_{s}
$$

To find the bounds of $1-\lambda_{1}$, first of all, the differential of $r / s_{2}$ is obtained by differentiating Eq. (4) as:

$$
\frac{d}{d t}\left(\frac{r}{s_{2}}\right)=\frac{-\sin \left(\theta_{s 2}\right)\left(\frac{r}{s_{2}}\right)}{\left(\frac{r}{s_{2}}\right)-\cos \left(\theta_{s 2}\right)} \dot{\theta}_{s}
$$

Substituting Eq. (43) and Eq. (39) into Eq. (32) gives:

$$
\begin{array}{r}
\frac{1}{\gamma}\left[\left(\frac{-\sin \left(\theta_{s 2}\right)\left(\frac{r}{s_{2}}\right)}{\left(\frac{r}{s_{2}}\right)-\cos \left(\theta_{s 2}\right)}\right) \mathbf{t}_{s}+\left(\frac{r}{s_{2}}\right) \mathbf{n}_{s}\right] \dot{\theta}_{s} \\
=-\left[1-\lambda_{1}\left(r / s_{2}, \theta_{s 2}\right)\right] \dot{\theta}_{s} \hat{\mathbf{n}}_{1}
\end{array}
$$

Taking a norm of both sides of this equation gives:

$$
\left|1-\lambda_{1}\right|=\frac{\frac{1}{\gamma}\left(\frac{r}{s_{2}}\right) \sqrt{\left(\frac{r}{s_{2}}\right)^{2}-2 \cos \left(\theta_{s 2}\right)\left(\frac{r}{s_{2}}\right)+1}}{\left|\left(\frac{r}{s_{2}}\right)-\cos \left(\theta_{s 2}\right)\right|} .
$$

Using Eq. (4) for the term under the square root of the above equation gives:

$$
\left|1-\lambda_{1}\right|=\frac{\left(\frac{r}{s_{2}}\right)}{\left|\left(\frac{r}{s_{2}}\right)-\cos \left(\theta_{s 2}\right)\right|} .
$$

Combining above equation and Eq. (6) gives:

$$
-\left(1+\frac{1}{\gamma}\right) \leq\left(1-\lambda_{1}\left(r / s_{2}, \theta_{s 2}\right)\right) \leq\left(1+\frac{1}{\gamma}\right)
$$

Then, the guidance command to UAV, turn rate $\dot{\theta}_{1}$, can be set as:

$$
\dot{\theta}_{1}=-\left(1+\frac{1}{\gamma}\right)\left|\dot{\theta}_{s}\right| \operatorname{sign}\left(\chi_{1}\right)-k_{\chi} \chi_{1}
$$

where control gain $k_{\chi}>0$ and

$$
\operatorname{sign}(x)=\left\{\begin{array}{lll}
1, & \text { if } & x>0 \\
0, & \text { if } \quad x=0 \\
-1, & \text { if } \quad x<0
\end{array}\right.
$$

The resulting Lyapunov rate is now:

$$
\begin{aligned}
\dot{L}_{1}= & \chi_{1}\left[-\left(1+\frac{1}{\gamma}\right)\left|\dot{\theta}_{s}\right| \operatorname{sign}\left(\chi_{1}\right)\right. \\
& \left.+\left(1-\lambda_{1}\right) \dot{\theta}_{s}\right]-k_{\chi} \chi_{1}^{2} \leq 0 .
\end{aligned}
$$

$\dot{L}_{1}$ is negative semi-definite since Eq. (47) makes an absolute magnitude of the first term in the square brackets equal to or greater than that of the second term. Thus the guidance law derived herein will produce a stable convergence of the UAV onto a rendezvous with the target. 


\section{Standoff target tracking case}

As the modified geometry for the standoff tracking problem was shown to be also invariant, its guidance law can be obtained in a similar way to the rendezvous case. Lyapunov function is identical as in Eq. (28) but using a different error angle function as:

$$
\chi_{1}=\left(\pi-\theta_{s m 1}\right)-\hat{\theta}_{s m 1}
$$

where $\left(\pi-\theta_{s m 1}\right)$ is the angle between the UAV velocity vector and the tangent line to the standoff circle around the target. In practice, an error angle $\chi_{1}$ can be obtained by using difference between the engagement vector $\hat{\mathbf{t}}_{1}$ from Eq. (13) and UAV's current tangent vector $\mathbf{t}_{1}$. Differentiating Eq. (13) with respect to time gives:

$$
\dot{\hat{\mathbf{t}}}_{1}=\frac{1}{\gamma}\left[\frac{d}{d t}\left(\frac{d_{r}}{s_{2}}\right) \mathbf{t}_{d}+\left(\frac{d_{r}}{s_{2}}\right) \dot{\mathbf{t}}_{d}\right]=\dot{\hat{\theta}}_{1} \hat{\mathbf{n}}_{1} .
$$

A geometric interpretation similar to the rendezvous problem is shown in Fig. 8. The same

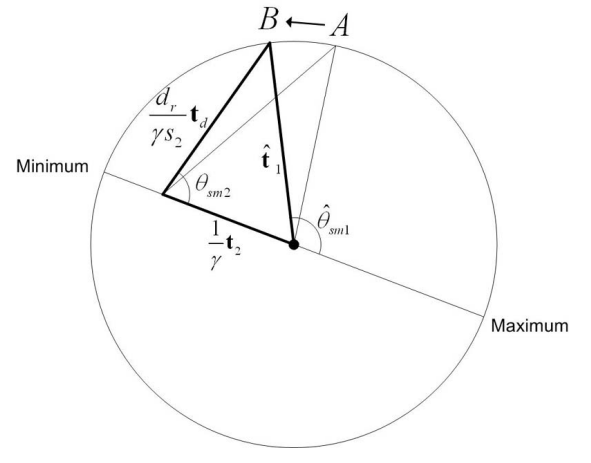

(a) Geometry $\gamma>1$

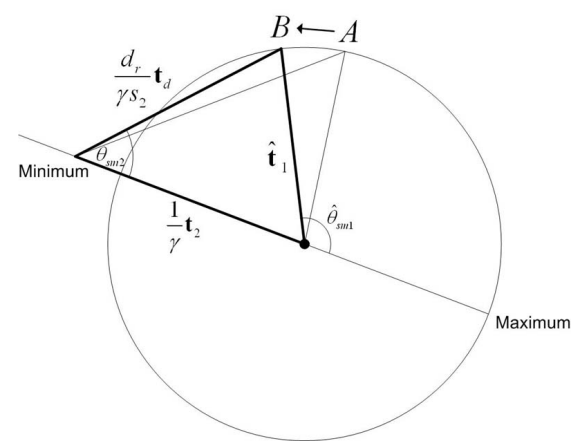

(b) Geometry $\gamma<1$

Fig. 8. Geometric interpretation of standoff tracking solutions

relation holds for the standoff tracking as for the rendezvous as:

$$
\dot{\hat{\theta}}_{s m 1}=\lambda_{1}\left(d_{r} / s_{2}, \theta_{s m 2}\right) \dot{\theta}_{s m 2}
$$

with:

$$
-\left(1+\frac{1}{\gamma}\right) \leq \lambda_{1}\left(d_{r} / s_{2}, \theta_{s m 2}\right) \leq\left(1+\frac{1}{\gamma}\right) .
$$

The differential of $r / s_{2}$ can be obtained by differentiating Eq. (21) as:

$$
\frac{d}{d t}\left(\frac{d_{r}}{s_{2}}\right)=\frac{-\sin \left(\theta_{s m 2}\right)\left(\frac{d_{r}}{s_{2}}\right)}{\left(\frac{d_{r}}{s_{2}}\right)-\cos \left(\theta_{s m 2}\right)} \dot{\theta}_{s m 2}
$$

In a similar way to the rendezvous case, by using $\theta_{d}, \frac{d_{r}}{s_{2}}$, and $\theta_{s m 2}$ instead of $\theta_{s}, \frac{r}{s_{2}}$, and $\theta_{s 2}$, the guidance command to UAV, turn rate $\dot{\theta}_{1}$, can be modified to:

$$
\dot{\theta}_{1}=-\left(1+\frac{1}{\gamma}\right)\left|\dot{\theta}_{d}\right| \operatorname{sign}\left(\chi_{1}\right)-k_{\chi} \chi_{1}
$$

where $k_{\chi}>0$. The resulting time-derivative of Lyapunov candidate is:

$$
\begin{aligned}
\dot{L}_{1}= & \chi_{1}\left[-\left(1+\frac{1}{\gamma}\right)\left|\dot{\theta}_{d}\right| \operatorname{sign}\left(\chi_{1}\right)+\right. \\
& \left.\left(1-\lambda_{1}\right) \dot{\theta}_{d}\right]-k_{\chi} \chi_{1}^{2} \leq 0 .
\end{aligned}
$$

To avoid the chattering problem which results from the discontinuity of sign function, continuous saturation function could be applied as given:

$$
\text { sat }\left(\frac{x}{\epsilon}\right)= \begin{cases}\frac{x}{\epsilon}, & \text { if }\left|\frac{x}{\epsilon}\right| \leq 1 \\ \operatorname{sign}\left(\frac{x}{\epsilon}\right), & \text { otherwise }\end{cases}
$$

where $\epsilon>0$ represents the width of the boundary layer.

Note that the LOS rate $\dot{\theta}_{s}$ to the target is replaced by the tangent line rate $\dot{\theta}_{d}$ to the standoff circle in Eq. (56). As the UAV sensor generally measures a LOS angle, a range and their rates, a calculation of the tangent line rate $\dot{\theta}_{d}$ is required starting from the following relation as:

$$
\theta_{d}=\theta_{s} \pm \theta_{d 1} .
$$

From Fig. 6, the following relation holds as:

$$
\tan \left(\theta_{d 1}\right)=\frac{d_{m}}{d_{r}}
$$

Differentiating both sides of the above equation gives

$$
\frac{1}{\cos \left(\theta_{d 1}\right)^{2}} \dot{\theta}_{d 1}=-\frac{d_{m}}{d_{r}^{2}} \dot{d}_{r}
$$


Rephrasing this yields:

$$
\dot{\theta}_{d 1}=-\frac{\cos \left(\theta_{d 1}\right)^{2} d_{m}}{d_{r}^{2}} \dot{d}_{r} .
$$

Substituting the relation $\cos \left(\theta_{d 1}\right)=\frac{d_{r}}{r}$ gives:

$$
\dot{\theta}_{d 1}=-\frac{d_{m}}{r^{2}} \dot{d}_{r} .
$$

Finally, substituting the above equation into the time-derivative of Eq. (59) yields:

$$
\dot{\theta}_{d}=\dot{\theta}_{s} \pm \frac{d_{m}}{r^{2}} \dot{d}_{r}
$$

Note that the solution involves the tangent line rate $\dot{d}_{r}$ to the standoff circle, not the LOS rate directly to the target position. This is practical since the range rate from the UAV to the target becomes zero and range measurement might be unobservable at the point of closest approach to the standoff orbit as the LOS gets normal to the tangent line.

In the case that the UAV is inside the standoff orbit, since a tangent line does not exist, the proposed guidance algorithm cannot be applied directly. Although several approaches can be suggested for this case such as keeping current speed until the UAV reaches standoff orbit or hybrid algorithm combining with other stable vector fields [13], this study uses modified control command $\dot{\theta}_{m}$ which exploits the condition of the UAV reaching the standoff orbit virtually whenever the UAV is inside the standoff orbit (i.e $d_{m}>r$ ) as:

$$
\dot{\theta}_{m}=\dot{\theta}_{1 t} \pm \Delta \dot{\theta} \tanh \left(\eta \frac{d}{d_{m}}\right)
$$

where $d=r-d_{m}$, and $\eta>0$ and $\Delta \dot{\theta}$ are control variables which adjust the convergence to the standoff orbit. $\dot{\theta}_{1 t}$ represents temporary control command which makes the UAV to track the target having the orbit radius of current distance from the target. As the UAV approaches to the desired standoff orbit, this modified control command gets closer to the original standoff tracking control command since $d$ goes to zero.

\section{NUMERICAL Simulations}

\section{A. Rendezvous and standoff tracking against constant-velocity target}

This section carries out numerical examples using the proposed differential geometric guidance for rendezvous and standoff tracking of a UAV against a moving ground target. Inhere, it is assumed that the target velocity is ideally available, there is no actuator delay in the UAV controller, and $w_{\max }$ is set to be $0.35 \mathrm{rad} / \mathrm{s}$. Firstly, Fig. 9 shows the simulation result of the rendezvous of the UAV to the target with different speed ratios between them. This simulation considers a UAV flying at different constant speeds of $\{10,20,30$, $40\} \mathrm{m} / \mathrm{sec}$ and a target traveling at a constant speed of $10 \mathrm{~m} / \mathrm{sec}$. Hence, $\gamma=1,2$, 3, or 4 for this case. As can be seen in Fig. 9, the faster the UAV is compared to the target, the closer a rendezvous position gets to the initial position of the target. The UAV with a higher $\gamma$ moves with a faster convergence time but consumes more control energy. For the case of the UAV travelling at 10 $\mathrm{m} / \mathrm{sec}(\gamma=1)$ distant from the target, a parallel solution results in no convergence to rendezvous since $r / s_{2} \rightarrow 0$ as shown in Fig. 10 .

The guidance law for standoff tracking is also applied to the scenario with the UAV flying different constant speeds of $\{20,30,40,50\} \mathrm{m} / \mathrm{sec}$ and a target travelling at a constant speed of 10 $\mathrm{m} / \mathrm{sec}$. Hence, $\gamma=2,3,4$, or 5 for this case. The cases of $\gamma=1$ or lower values of $\gamma$ are dropped as the UAV not faster than the target is difficult to catch up with a standoff circle continuously. The required standoff distance from the UAV to the target $d_{m}$ is set to be $300 \mathrm{~m}, w_{\max }$ is set to be $0.3 \mathrm{rad} / \mathrm{s}$, and the rotating direction of the UAV with respect to the target position is clockwise. The resultant trajectories for different $\gamma$ are shown in Fig. 11. Figure 12 shows the standoff tracking for UAVs inside the standoff orbit with $d_{m}=400$ $\mathrm{m}, \Delta \dot{\theta}=0.1$ and $\eta=5$. As can be seen in the trajectories and relative distances of Fig. 11-12, the faster the UAV is compared to the target, the faster it converges to the standoff circle around the target. However, the UAV with a higher $\gamma$ moves with consuming more control energy and aggressive manoeuvres.

\section{B. Standoff tracking against realistic movement of ground vehicle}

To verify the feasibility and benefits of the proposed approach in this paper, a differential geometric guidance algorithm is applied to a civilian 


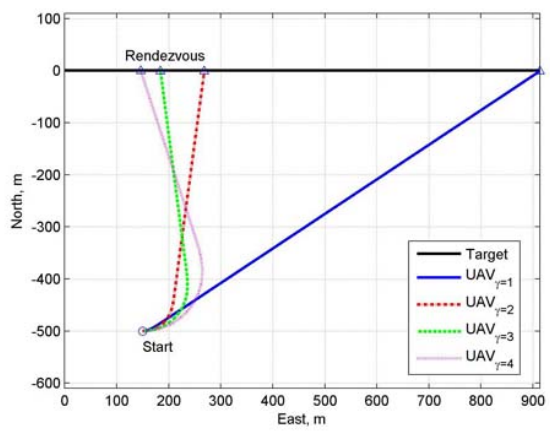

(a) Trajectory

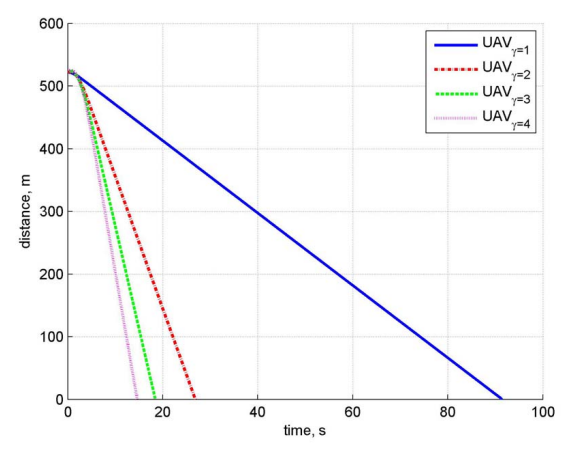

(b) Relative distance

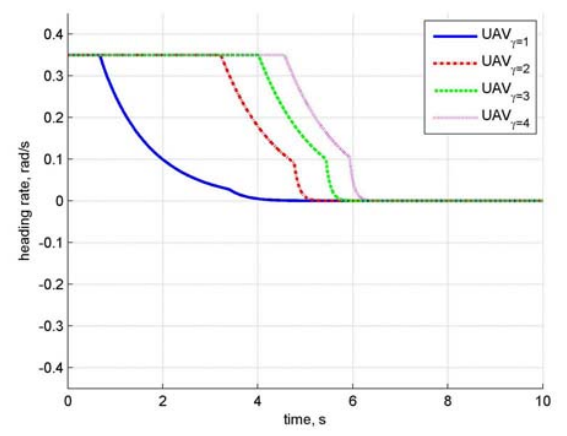

(c) Heading rate

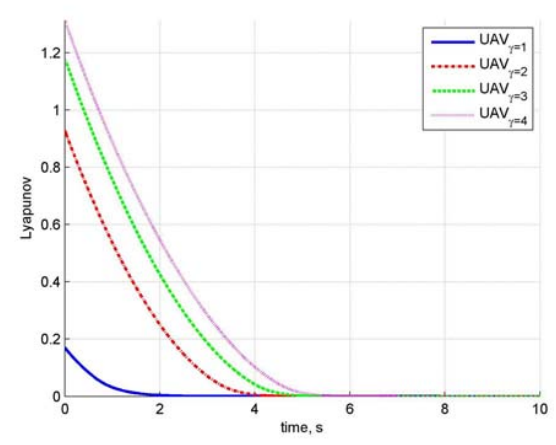

(d) Lyapunov function

Fig. 9. Rendezvous of UAV to target with different speed ratios: $\gamma=1,2,3$, and 4

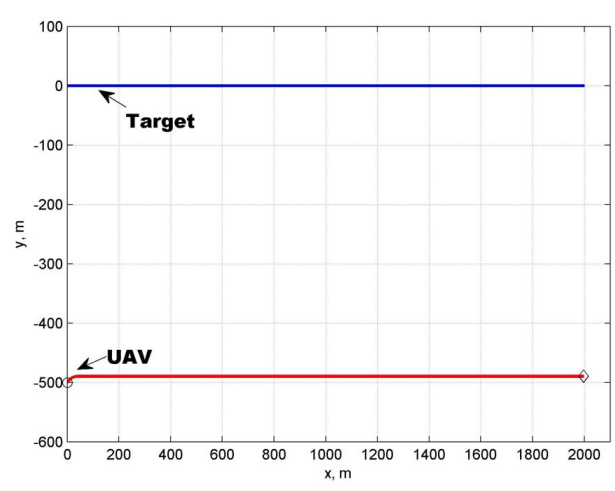

Fig. 10. Parallel solution of rendezvous of the UAV to the target

ground target tracking scenario using two cooperative UAVs. For this, the vehicle trajectory data acquired at $2 \mathrm{~Hz}$ in a S-Paramics [19] traffic model of Devizes, Wiltshire, United Kingdom, is used to generate the MTIR (Moving Target Indicator Radar) measurements composed of relative range and azimuth angle with respect to a position of the UAV as shown in Fig. 13. The ground vehicle departs at the western side of Devizes and traverses a part of the town centre and then turns back until the journey ends at the northwestern side of Devizes [20]. As shown in Figs. 13 14, the ground vehicle moves complicatedly with frequently changing direction of driving and its speed.

1) Tracking filter for target localisation and prediction: After analyzing the car trajectory data acquired by running a $\mathrm{S}$-Paramics traffic program and general driving behaviours, it is observed that the jerk of the car is not negligible, but the acceleration is piecewise constant for a specific duration of time. This study, therefore, considers acceleration dynamics [20], [21] to apply it to tracking of the moving ground vehicle. The MTIR radar measurement $(r, \phi)^{T}$ can be defined as the following nonlinear relation using the target position $\left(x_{k}^{t}, y_{k}^{t}\right)^{T}$ and the UAV position $\left(x_{k}, y_{k}\right)^{T}$ as:

$$
\begin{aligned}
\mathbf{z}_{k} & =\left[\begin{array}{l}
r_{k} \\
\phi_{k}
\end{array}\right]=h\left(\mathbf{x}_{k}^{t}\right)+\nu_{k} \\
& =\left(\begin{array}{c}
\sqrt{\left(x_{k}^{t}-x_{k}\right)^{2}+\left(y_{k}^{t}-y_{k}\right)^{2}} \\
\tan ^{-1} \frac{y_{k}^{t}-y_{k}}{x_{k}^{t}-x_{k}}
\end{array}\right)+\nu_{k}
\end{aligned}
$$




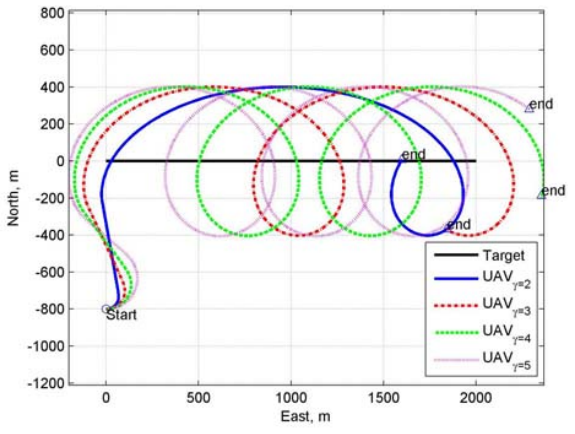

(a) Trajectory

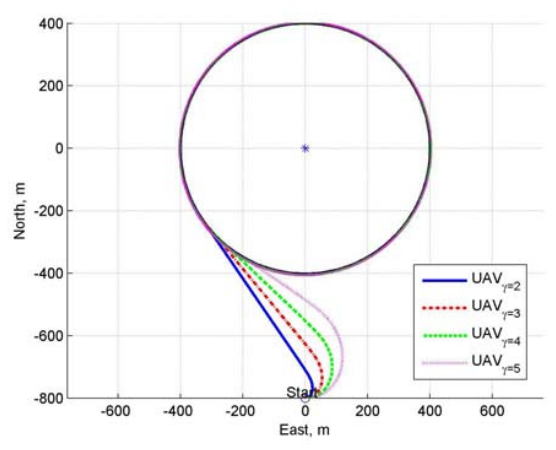

(b) UAV trajectory relative to target

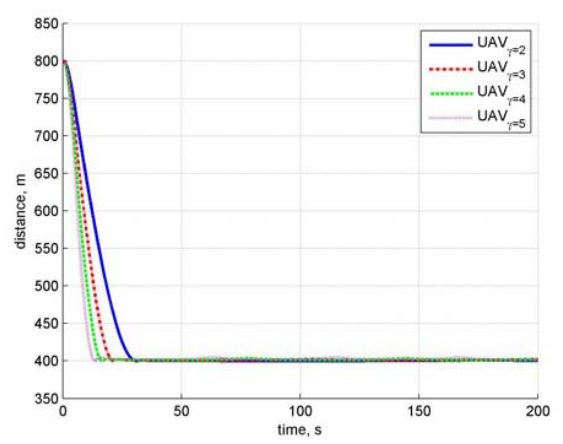

(c) Relative distance

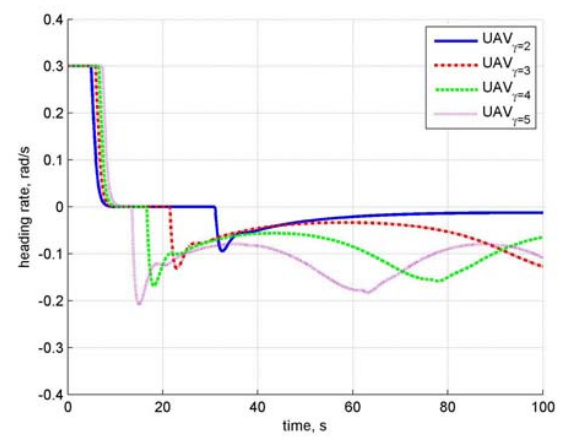

(d) Heading rate

Fig. 11. Standoff tracking of the UAV to the target with different speed ratios between UAV and target: $\gamma=2,3,4$, and 5

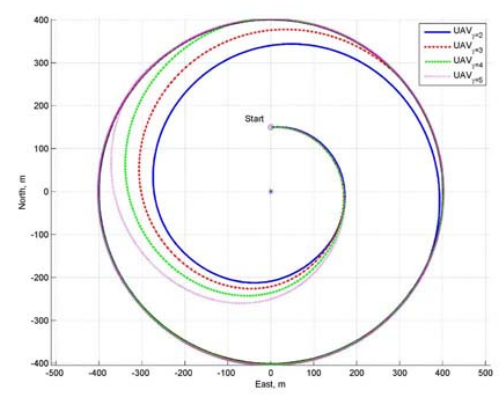

(a) UAV trajectory relative to target

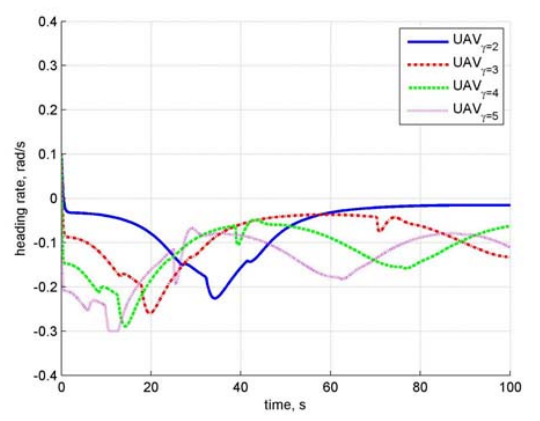

(b) Heading rate

Fig. 12. Standoff target tracking of the UAV inside standoff orbit, $\Delta \dot{\theta}=0.1$ and $\eta=5$

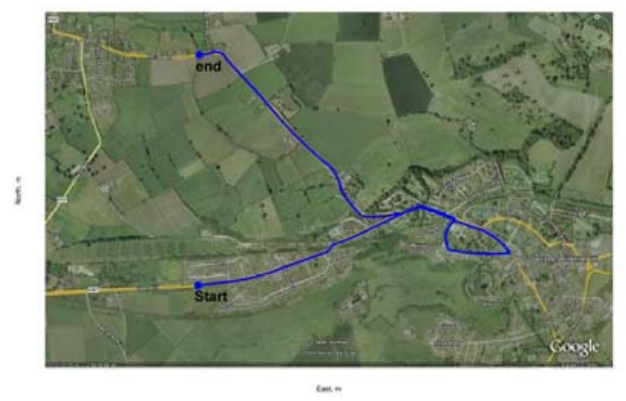

Fig. 13. Ground Vehicle trajectory within the road network of Devizes, Wiltshire, United Kingdom with GIS satellite data overlaid thanks to Google Map

where $\nu_{k}$ is a measurement noise vector, and its noise covariance matrix is defined as:

$$
V\left[\nu_{k}\right]=R=\left[\begin{array}{cc}
\sigma_{r}^{2} & 0 \\
0 & \sigma_{\phi}^{2}
\end{array}\right] .
$$

Since this study assumes multiple UAVs carry out the cooperative standoff tracking of a ground moving target, each UAV's MTIR sensor can get its own measurement and execute the tracking filter algorithm separately. After each UAV receives the other's estimation via communication link, it can run a decentralised sensor fusion to enhance the 
tracking accuracy. Considering the fact that $h\left(\mathbf{x}_{k}^{t}\right)$ in the measurement equation is nonlinear as shown in Eq. (66) and the advantage of using information form in multi-sensor system with its less communication load, the localisation of a target is designed by the extended Information filter (EIF) [22] as:

Prediction

$$
\begin{gathered}
\mathbf{y}_{k \mid k-1}^{t}=Y_{k \mid k-1} F_{k} Y_{k-1 \mid k-1}^{-1} \mathbf{y}_{k-1 \mid k-1}^{t} \\
Y_{k \mid k-1}=\left(F_{k} Y_{k-1 \mid k-1}^{-1} F_{k}^{T}+Q_{k}\right)^{-1}
\end{gathered}
$$

Update

$$
\begin{aligned}
\mathbf{y}_{k \mid k}^{t}= & \mathbf{y}_{k \mid k-1}^{t}+H_{k}^{T}\left(R_{k}\right)^{-1} \cdot \\
& {\left[\mathbf{z}_{k}-h\left(\mathbf{x}_{k \mid k-1}^{t}\right)+H_{k} \mathbf{x}_{k \mid k-1}^{t}\right] } \\
Y_{k \mid k}= & Y_{k \mid k-1}+H_{k}^{T}\left(R_{k}\right)^{-1} H_{k}
\end{aligned}
$$

where $Y_{k}=\left(P_{k}\right)^{-1}$ and $\mathbf{y}_{k}^{t}=Y_{k} \mathbf{x}_{k}^{t}$ represent the information matrix and information state vector, respectively. And the output matrix $H_{k}$ is a Jacobian of $h$ with respect to the time-update state $\mathbf{x}_{k \mid k-1}^{t}$. Moreover, this study adopts the decentralised EIF [22] scheme to fuse communicated data under the assumption that the communication bandwidth is wide enough to transmit information in both directions between a pair of UAVs.

2) Standoff tracking results: To maximise sensor coverage to the target, enhance the accuracy of the target estimation or avoid the collision, the phase coordination between UAVs is required during the mission. In this scenario, the phaseangle keeping is accomplished separately from the standoff orbit tracking by controlling the speed of UAVs as [5], [6], [12]:

$$
u_{v}= \pm k_{v}\left(\Delta \theta-\theta_{d}\right) d_{m}+v_{d}
$$

where $k_{v}>0$ is a control gain, $\Delta \theta$ is a difference of azimuth angles between UAVs relative to the target position, $\theta_{d}=\pi$ is a desired phase difference between UAVs, and $v_{d}$ is a desired velocity command. In other words, by changing the speed of two vehicles accordingly moving on the same standoff orbit, desired angular separation can be achieved while avoiding collision each other as well as maximise the sensor coverage to the target. The setting of parameters needed for the proposed differential geometry guidance can be found in Table I. Firstly, the decentralised extended Information filter shows a good tracking accuracy
TABLE I

SIMULATION PARAMETERS

\begin{tabular}{llc}
\hline Parameter & Value & Unit \\
\hline \hline$\theta_{d}$ & $\pi$ & $\mathrm{rad}$ \\
$v_{d}$ & 40 & $\mathrm{~m} / \mathrm{s}$ \\
$d_{m}$ & 500 & $\mathrm{~m}$ \\
$v_{\max }$ & 10 & $\mathrm{~m} / \mathrm{s}$ \\
$\omega_{\max }$ & 0.2 & $\mathrm{rad} / \mathrm{s}$ \\
$\tau_{v}, \tau_{\omega}$ & $1 / 3$ & $\mathrm{sec}$ \\
$\left(k_{v}, k_{\chi}\right)$ & $(0.1,1)$ & $\mathrm{N} / \mathrm{A}$ \\
$\left(\sigma_{r_{1}}, \sigma_{r_{2}}\right)$ & $(5,7)$ & $\mathrm{m}$ \\
$\left(\sigma_{\phi_{1}}, \sigma_{\phi_{2}}\right)$ & $(3.5,2)$ & $\mathrm{deg}$ \\
$(\Delta \dot{\theta}, \eta)$ & $(0.1,5)$ & $\mathrm{N} / \mathrm{A}$ \\
\hline
\end{tabular}

even in $\mathrm{x}$ and $\mathrm{y}$ coordinate velocity as shown in Fig. 14. Figure 15(a) displays the absolute trajectories of UAVs and ground vehicle, and Fig. 15(b) displays the relative trajectories of UAVs with respect to the ground vehicle. As can be seen in Figs. 15(c) $(\mathrm{d})$, the proposed guidance shows a reasonably good tracking performance for both the standoff distance error and phase angle keeping between UAVs. Figures 15(e) (f) show the control input histories of speed and turn rate. These are commanded control inputs, and as can be seen in the UAV dynamic model as Eq. (22), first-order time constants for considering actuator delay are used to respect dynamic constraints in the simulation providing smooth and realistic flying path. Moreover, standoff tracking performance with different control gain $k_{\chi}$ and velocity ratio $\gamma$ is investigated using the same scenario as above, but with a single UAV having a constant velocity during each simulation as shown in Fig. 16. In this figure, $\gamma_{\text {mean }}$ represents the mean value of velocity ratio between the UAV and the moving target of each simulation, and it is changed by using different velocities of the UAV with the same ground target. Note that the variable $\gamma$ is not tuned but in real-time computed in the guidance loop using the estimated target speed and the UAV's own one. As velocity ratio $\gamma_{\text {mean }}$ and the control gain $k_{\chi}$ increase, the standoff distance error decreases except for the case of a small control gain. Since the target keeps changing its velocity, it is difficult for the UAV using a small control gain to track the target precisely, and it is becoming more difficult with increasing velocity of the UAV. On the other hand, the control effort obtained 


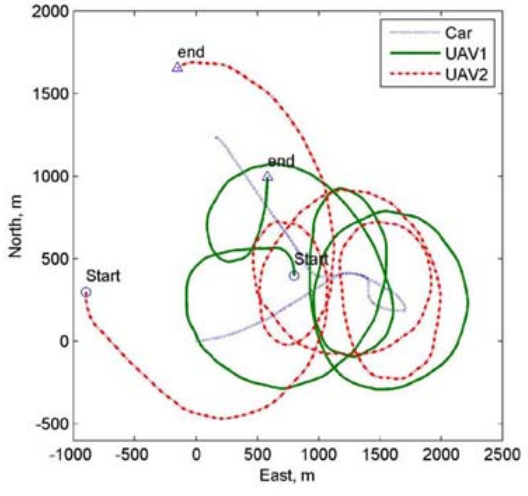

(a) Absolute trajectories of UAVs and target

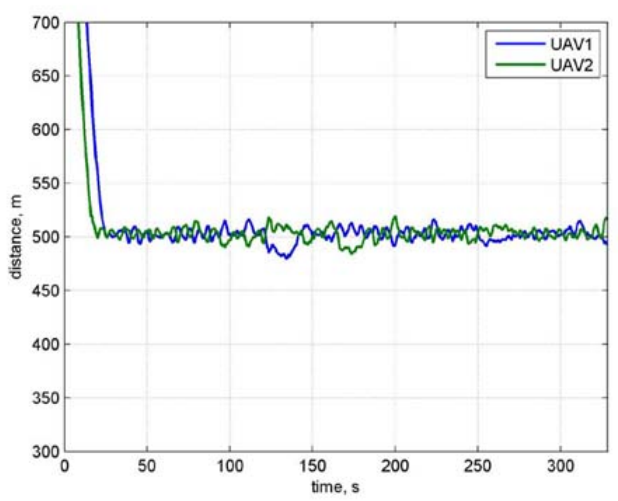

(c) Standoff distance error

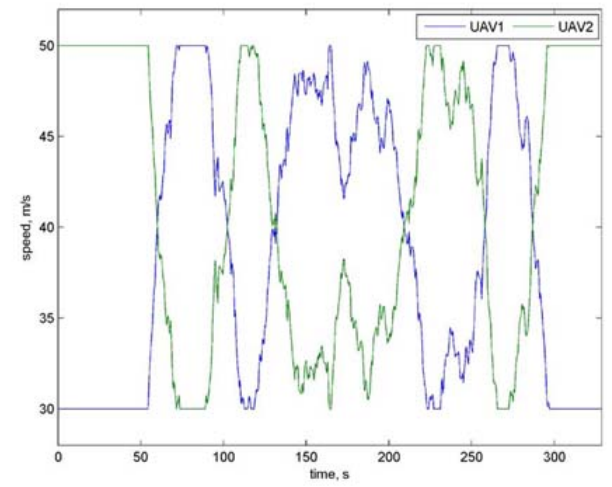

(e) Control input of UAVs: $u_{v}$

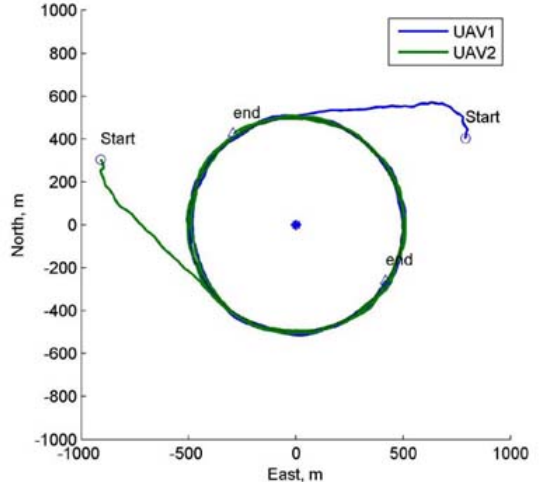

(b) Relative trajectories of UAVs with respect to target

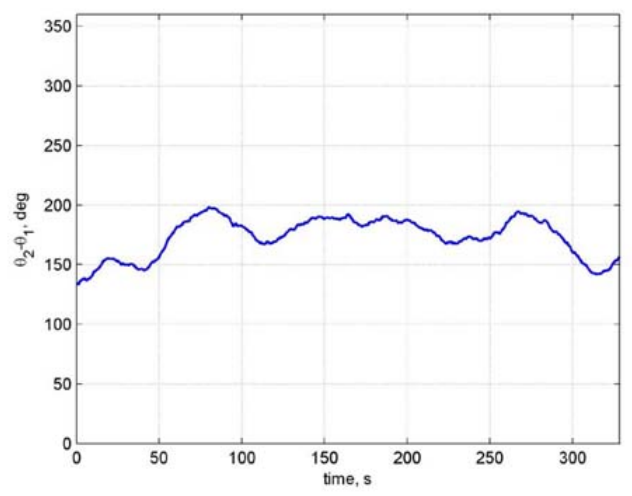

(d) Phase angle difference of UAVs

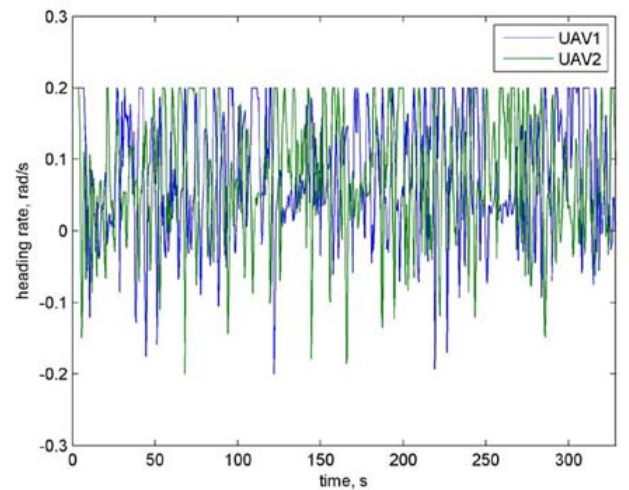

(f) Control input of UAVs: $u_{\omega}$

Fig. 15. Standoff tracking simulation results 


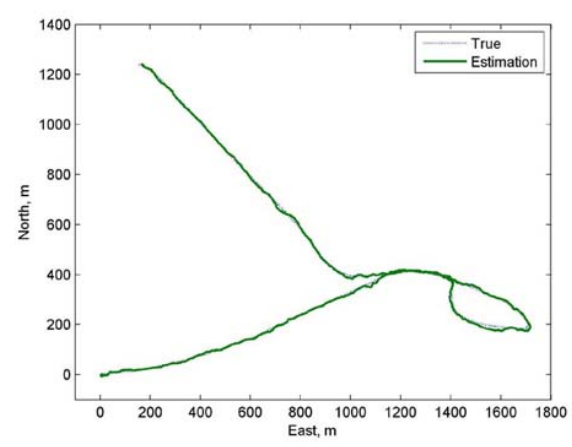

(a) Trajectory
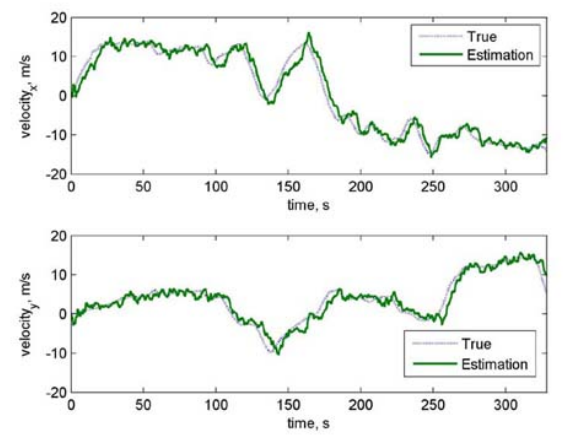

(b) $\mathrm{x}$ and $\mathrm{y}$ velocity

Fig. 14. Ground target estimation using DEIF

by integrating the time histories of $\left|u_{w}\right|$ tends to increase as the control gain and velocity ratio increase continuously. In short, these simulation results show the trade-off between tracking error and control effort, and facilitate system operators to define a requirement of the speed dominance of UAVs over the target depending on the mission specification.

\section{CONClusion}

This paper presented a novel guidance algorithm ensuring rendezvous and standoff tracking against a moving target using differential geometry. Using the relative geometry between the UAV and the target, convergent, divergent and parallel solutions were obtained depending on the initial positions and velocity magnitude ratio. Then, the convergent solution which gives the required tangent direction was used to guide UAV onto the rendezvous or standoff tracking geometry. The proposed guidance law showed a reasonable tracking performance for the realistic civilian ground target tracking scenario having the advantage of simple stability analysis

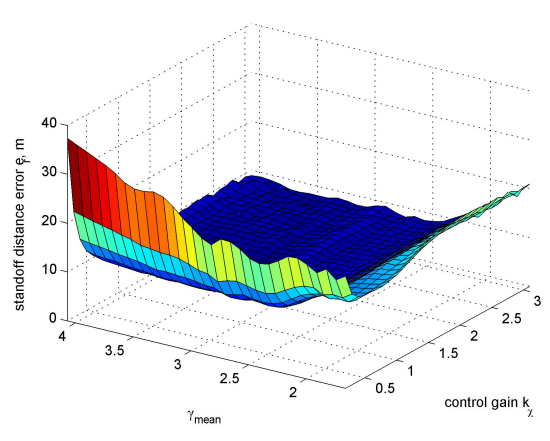

(a) Standoff distance error, $e_{r}=r-d_{m}$

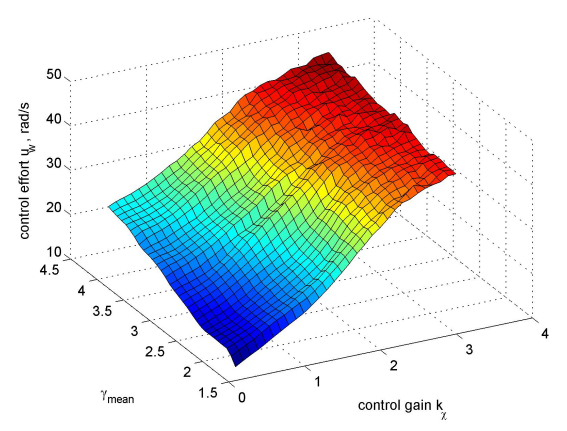

(b) Control effort, $u_{w}$

Fig. 16. Standoff tracking performance with different control gain $k_{\chi}$ and velocity ratio $\bar{\gamma}$

by using geometric relation and Lyapunov theory as well as less tuning parameters. As a future work, the proposed differential geometric approach will be extended to consider the target manoeuvre with unknown or imperfect information by adding robust or adaptive control term. The control of multiple patterns using the proposed differential geometric concept and a comparison with other comprehensive approaches [23], [24] will also be followed for cooperative missions.

\section{REFERENCES}

[1] S. Martinez and F. Bullo. Optimal sensor placement and motion coordination for target tracking. Automatica, 42(4):661668, 2006.

[2] G. Gu, P. Chandler, C. Schumacher, A. Sparks, and M. Pachter. Optimal cooperative sensing using a teams of UAVs. IEEE Transactions on Aerospace and Electronic Systems, 42(4):1446-1458, 2006.

[3] E.W. Frew. Sensitivity of cooperative target geolocalization to orbit coordination. Journal of Guidance, Control, and Dynamics, 31(4):1028-1040, 2008.

[4] D.A. Lawrence. Lyapunov vector fields for UAV flock coordination. 2nd AIAA Unmanned Unlimited conference, workshop, and exhibit, Reston, VA, USA, 2003. 
[5] E.W. Frew, D.A. Lawrence, and S. Morris. Coordinated standoff tracking of moving targets using lyapunov guidance vector fields. Journal of Guidance, Control, and Dynamics, 31(2):290-306, 2008.

[6] S. Morris and E.W. Frew. Cooperative tracking of moving targets by teams of autonomous unmanned air vehicles. Technical Report FA9550-04-C-0107, MLB Company, 2005.

[7] T.H. Summers, M.R. Akella, and M.J. Mears. Coordinated standoff tracking of moving targets: Control laws and information architectures. Journal of Guidance, Control, and Dynamics, 32(1):56-69, 2009.

[8] D. Kingston and R. Beard. UAV spaly state configuration for moving targets in wind. Lecture Notes in Control and Information, 369:109-128, 2007.

[9] H. Chen, K. Chang, and C. Agate. Tracking with UAV using tanget-plus-lyapunov vector field guidance. International Conference on Information Fusion, Seattle, WA, USA, 2009.

[10] R. Sepulchre, D.A. Paley, and N.E. Leonard. Stabilization of planar collective motion: All-to-all communication. IEEE Transactions on Automatic Control, 52(5):811-824, 2007.

[11] D.J. Klein and K.A. Morgansen. Controlled collective motion for trajectory tracking. In American Control Conference, American Control Conference, Minneapolis, Minnesota, 2006.

[12] R.A. Wise and R.T. Rysdyk. UAV coordination for autonomous target tracking. AIAA Guidance, Navigation and Control Conference, Keystone, Colorado, USA, 2006.

[13] C.G. Prevost, O. Theriault, A. Desbiens, and E. Poulin. Receding horizon model-based predictive control for dynamic target tracking: a comparative study. AIAA Guidance, Navigation, and Control Conference, Chicago, Illinois, USA, 2009.

[14] S. Kim, H. Oh, and A. Tsourdos. Nonlinear model predictive coordinated standoff tracking of moving ground vehicle. Journal of Guidance, Control, and Dynamics, In press, 2012.

[15] B.A. White, R. Zibkowski, and A. Tsourdos. Direct intercept guidance using differential geometry concepts. IEEE Transactions on Aerospace and Electronic Systems, 43(3):899-919, 2007.

[16] B.A. White, H.S. Shin, and A.Tsourdos. UAV obstacle avoidance using differential geometry concepts. In 18th IFAC World Congress, Milano, Italy, Aug 2011.

[17] D.A. Lawrence, E.W. Frew, and W.J. Pisano. Lyapunov vector fields for autonomous unmanned aircraft flight control. Journal of Guidance, Control, and Dynamics, 31(5):1220 1229, 2008.

[18] B. O'Neill. Elementary Differential Geometry (2nd ed.). San Diego, CA: Academic Press, 1997.

[19] SIAS Limited. S-Paramics software. http://www.sias.com, Jan 2011.

[20] S. Kim, R.W. Zbikowski, A. Tsourdos, and B.A. White. Behaviour recognition of ground vehicle for airborne monitoring by UAV swarm. IFAC Symposium on Intelligent Autonomous Systems, Lecce, Italy, 2010.

[21] K. Mehrotra and P.R. Mahapatra. A jerk model for tracking highly maneuvering targets. IEEE Transactions on Aerospace and Electronic Systems, 33(4):1094-1105, 1997.

[22] A.G.O. Mutambara. Decentralized Estimation and Control for Multisensor Systems. CRC Press LLC, Boca Raton, Florida, 1998.

[23] A.P. Aguiar, I. Kaminer, R. Ghabcheloo, A.M. Pascoal, E. Xargay, N. Hovakimyan, C. Cao, and V. Dobrokhodov. Coordinated path following of multiple UAVs for time-critical missions in the presence of time-varying communication topologies. In 17th IFAC World Congress, Seoul, Korea, July 2008.

[24] M. Shanmugavel, A. Tsourdos, B. A. White, and R. Zbikowski. Differential geometric path planning of multiple UAVs. Journal of Dynamic Systems, Measurement, and Control, 129:620-632, 2007. 